\title{
ESCENARIOS PROBLEMATIZADORES DE LAS ORGANIZACIONES EDUCATIVAS DESDE LA PERSPECTIVA DEL DESARROLLO DE COMPETENCIAS EMOCIONALES EN LOS DOCENTES EN FORMACIÓN
}

\author{
TROUBLESOME SCENARIOS WITHIN EDUCATIONAL INSTITUTIONS FROM THE \\ PERSPECTIVE OF EMOTIONAL COMPETENCES DEVELOPMENT \\ OF IN-TRAINING TEACHERS
}

\author{
Olga Cleosilda Chica-Palma* y Jorge Oswaldo Sánchez-Buitrago**
}

\begin{abstract}
RESUMEN
En el marco de la investigación titulada "Las organizaciones educativas como escenarios para el desarrollo de competencias emocionales: una lectura comprensiva de la formación de docentes en las Escuelas Normales Superiores de Colombia", se configuraron cuatro escenarios comprensivos que evidencian serias problemáticas de las organizaciones educativas, asociadas a la formación emocional. En este artículo se sustentan cuatro escenarios problematizadores claves, a saber: el primero, la nueva estructura y condición familiar que no aporta los patrones emocionales necesarios para una formación integral de calidad; el segundo, la emergencia de una nueva condición de lo humano que está demandando nuevas respuestas de las organizaciones formadoras de maestros; el tercero, las nuevas realidades y desafíos que impone la sociedad y los medios de comunicación a la formación emocional; y el cuarto, las dificultades y retos para la formación docente, en particular en el ámbito de las emociones. Lo anterior, en perspectiva de aportar a la incorporación sistemática del desarrollo de competencias emocionales en las propuestas formativas de los docentes; lo que ha de contribuir a una mejora de la calidad educativa y en especial de las condiciones y posibilidades del ejercicio de la profesión docente.
\end{abstract}

Palabras Clave: Competencias emocionales, calidad educativa, organizaciones educativas, gestión educativa.

Fecha de recepción: Junio 16 de 2015 / Fecha de aceptación: Agosto 03 de 2015

Tipología: Artículo de Investigación Científica y Tecnológica

Para citar este artículo: Chica, P. 0., \& Sánchez, B. J. (2015). Escenarios problematizadores de las organizaciones educativas desde la perspectiva del desarrollo de competencias emocionales en los docentes en formación. Praxis. Vol. 11, 116 - 131

\footnotetext{
*Candidata a Doctora en Ciencias de la Educación de la Universidad del Magdalena-RUDECOLOMBIA. Coordinadora Académica de la Escuela Normal Superior San Pedro Alejandrino, de Santa Marta y Docente Catedrática de la Facultad de Ciencias Educación de la Universidad del Magdalena. Colombia. Email: olgacleo@gmail.com

**Doctor en Educación (Universidad de Salamanca, España). Docente de Planta de la Facultad de Educación de la Universidad del Magdalena, adscrito a la Facultad de Ciencias de la Educación de la Universidad del Magdalena. Investigador Asociado, clasificado por COLCIENCIAS/2015. Colombia. Email: joswaldosanchez@gmail.com
} 


\section{ABSTRACT}

As part of the research entitled "Educational Intitutions conceived as scenarios for the development of emotional competences: a comprehensive reading based on the training of teachers in Escuelas Normales Superiores from Colombia," four comprehensive scenarios were configured which unveil compromising issues of the educational organizations, in relation to emotional training. Four key troublesome scenarios are supported in the present paper, namely, the first one, the new structure and familiar condition that does not provide the necessary emotional models for a qualified integral training; the second one, the emergency boosted from a new human condition that is requiring new answers regarding to the teacher-training organizations; the third one, the new realities and challenges imposed by both society and mass media over the emotional training, and the fourth one, the difficulties and challenges within teaching training, particularly connected with emotions. All the above, is aimed to support the systematic incorporation of the emotional competences development within the formative proposals of teachers; intended to contribute to an educational quality improvement and especially enhance the conditions and possibilities of the teaching profession execution.

Keywords: Emotional competences, educational quality, educational organizations, education management.

\section{INTRODUCCIÓN}

$\mathrm{E}$ el contexto de una época signada por grandes cambios, la sociedad ha venido enfrentando al sistema educativo a una dinámica de transformaciones constantes derivadas de múltiples fenómenos sociales: la violencia estructural, la desintegración familiar, la sociedad de la información, el incremento vertiginoso de la ciencia y la tecnología, la universalización de los mercados, la globalización de la economía, la identidad de la aldea global, la incorporación de las tecnologías de la información y la comunicación a la vida cotidiana, la sociedad de consumo, la multiculturalidad y muchos otros fenómenos más que están enfrentado a las organizaciones educativas y en ellas a los docentes, al reto de un ejercicio profesional cada vez más complejo; que exige de ellos competencias muy divesrsas para responder a una sociedad compleja y en cosntante cambio.

Pero este cambio ha sido tan vertiginoso que las organizaciones educativas no han alcanzado a responder y ha llevado a la escuela a desfasarse de las necesidades de su contexto. Todo esto agravado por una dinámica familiar que como escenario primordial de la sociedad ha mutado hacia dinámicas cada vez menos estructuradas y hacia patrones de socialización más inestables. Si bien siguiendo los aportes de Sánchez (2009), la educación se asume como un proceso continuo y permanente de complejización del individuo y de la sociedad, con una doble función de preservación y transformación de la cultura, lo cual implica una relación dialéctica e histórica entre el colectivo social y los individuos, en un constante proceso de cambio y evolución que los configura mutuamente. Esto implica que tanto la sociedad como la educación deben avanzar a un ritmo acompasado que le permita a cada una reconfigurarse con la otra.

En esta perspectiva, con base en Sánchez (2010) las organizaciones educativas deben asumirse como sistemas autopoiéticos y autorreferenciales que tienen como misión ofrecer y/o gestionar procesos educativos que interpelan y promueven el desarrollo de los individuos y de la sociedad, desde la perspectiva de sus múltiples aprendizajes.

Pero la dinámica social ha superado a la dinámica reconstitutiva de la educación, generando en las organizaciones educativas incertidumbre y perplejidad manifestada en un proceso de marginación de la realidad generando una ruptura entre las necesidades de la sociedad y 
las respuestas brindadas desde la educación. Esta ruptura entre la realidad social y las organizaciones educativas según Castro $^{1}$ (2012) se manifiesta en una doble marginación:

- “Una marginación externa, motivada por el desinterés de los educandos que cotidianamente viven en un mundo cada vez más rápido, en el proceso de enseñanza-aprendizaje y de la comunidad donde éste se inserta, luego de percatarse que las organizaciones educativas no estaban cubriendo las necesidades requeridas por la sociedad.

- Y la marginación interna, motivada por el distanciamiento entre la rapidez de la sociedad y la lentitud de la organización educativa por alcanzarla; lo que genera en las organizaciones un rechazo al cambio impuesto desde el exterior." (p. 84)

Estas manifestaciones de marginación se expresan en múltiples fenómenos epistémicos y empíricos que desde los planteamientos de este capítulo constituyen el problema central de las organizaciones educativas en la actualidad, propiciando que frente a las necesidades de la complejidad de hoy la organización educativa se ha estancadotransitando por caminos diferentes a los que requiere la sociedad: "el estancamiento de la interrelación con el medio ambiente, comenzó a envolver a las organizaciones, sumergiéndolas en un limbo hermético e impenetrable al entorno" (Castro, 2012, p. 84), haciendo necesario desarrollar procesos que la dinamicen y acompasen con la sociedad que le ha correspondido educar.

Esta dinámica de marginación de las organizaciones educativas se manifiesta a través de muchos fenómenos, uno de ellos retomando los aportes desde la teoría de la complejidad, se argumenta que una de las causas determinantes es la visión simplificante que subyace en la realidad de estas organizaciones; fundada en un paradigma lineal y disyuntivo que según Morín (2001) tiende a aislar en lugar de integrar, que tiende a reducir

1. Bernardo Alonso Castro Sáez, Dr. en Ciencias de la Educación . Asesor Técnico Pedagógico en Ministerio de Educación de Chile. Académico en la Facultad de Educación de la Universidad Austral de Chile. desde una racionalidad lineal y de abstracción, que se contenta con establecer leyes generales desconociendo las particularidades de dónde surgen.

Esta visión simplificada, que surge a partir de la ciencia clásica y que aparece con la modernidad, fue muy exitoso a partir de la afirmación de que la naturaleza es objetiva, es decir, externa al hombre, que es el sujeto capaz de conocerla y dominarla en su propio beneficio, pero su utilidad y uso en las organizaciones educativas las dejó rezagadas del tránsito veloz de la postmodernidad. Este paradigma le otorgó a las organizaciones un papel replicador del sistema social, estableciendo de manera clara las regularidades que debían ser perpetuadas e imposibilitándole reconocer los cambios de su entorno. Según Castro, creando un sistema defensivo que la hace detener las transformaciones y evitar los cambios: "la organización educativa se vio desbordada, al utilizar un sistema fragmentario proveniente de una concepción científico-racional, que al no entender la movilidad ricamente creativa de la realidad" (2012, p. 87), termina negando la propia esencia creativa, cambiante y compleja del individuo, asumiéndolo desde una perspectiva unidimensional, aislado de su realidad.

Desde esta visión simplificada y reduccionista la organización educativa restringió su labor a lo cognitivo, negando el espacio formativo a dimensiones tan vitales como lo ético, los estético, lo corporal y lo emocional, entre otras.

Quiza una de las más excluidas y que hoy la familia y la sociedad reclama con mayor urgencia a la organización educativa es la dimensión emocional. La realidad simplificadora de la educación actual la ha desterrado del ámbito escolar, pero la nueva perspectiva educativa compleja la requiere y la reclama; para ello es necesario visibilizarla y hacerla evidente en la naturaleza de la organización como constructo social complejo. Para ello se requiere que cada uno de los actores de la organización educativa supere el pensamiento del antiguo paradigma, pero no solo en la dimensión externa de lo educativo, sino en la dimensión interna como personas; lo que implica concebirse cada uno a su vez como sujeto complejo, multidimensional 
cognitivo, social, cultural, político, espiritual, emocional... La emocionalidad se asume como una dimensión constitutiva de la organización en la que igualmente es necesario generar espacios de desarrollo y gestión institucional; porque "enseñar es un acto emocional por acción o por omisión, por diseño o por defecto" (Extremera y Fernandez, 2005, p. 497).

En este contexto las organizaciones educativas formadoras de docentes, inmersas igualmente en esta misma problemática del paradigma simplificador, han caracterizado los procesos educativos de las nuevas generaciones de docentes por procesos igualmente simplificadores y disyuntivos donde la prioridad ha sido el conocimiento disciplinar en detrimento de otras competecias, incluyendo las emocionales, tan necesarias en los maestros de hoy;

si observamos la estructura de las diferentes formaciones ofrecidas a nuestros docentes, podemos llegar a la conclusión de que se presupone que éstos no necesitan nada más que un conjunto de teorías, métodos y técnicas científicas que deberán aplicar sobre sus alumnos y que garantizan su buen hacer, ignorando así su condición de ser social y emocional. (Fernandez, Palomero y Teruel, 2009, p. 146)

De esta manera la sociedad exige a la nueva generación de maestros la ejecucución de procesos que inclusive ellos mismos no han tenido posibilidad de desarrollar o trabajar como objeto de estudio.

Asumir entonces a la organización educativa formadora de maestros, en este caso las Escuelas Normales, desde este nuevo paradigma de la complejidad tiene una doble implicación; la primera, ofrecer una educación integral para las personas que la componen y en segunda instancia implica entender el reto de asumir su condición de maestros en formación, los cuales requieren además de su formación individual, una formación que los haga competentes para formar a otros. Desde esta lógica pensar en la cualificación de este tipo de organizaciones educativas es importante para el sistema educativo en general ya que el impacto de su calidad sería mucho mayor, dado que aportaría no solo al proceso educativo de los actores que la componen sino a su vez contribuiría a los procesos de calidad de las organizaciones educativas de las cuales harán parte en el futuro.

La formación docente es un campo estratégico de la educación actual, ya que crea un espacio de posibilidad para la transformación del quehacer docente, del vínculo pedagógico y de la gestión e institucionalidad educativa. Esta afirmación se sustenta, a su vez, en el reconocimiento del papel estratégico que juega el profesor en las transformaciones educativas. (Messina, 1999, pág. 92)

Desde este contexto y a partir de los elementos empiricos de esta problematizacón, se reconoce que la labor educativa cotidiana de los maestros exige altas dosis de desgaste emocional; enfermedades como el sindrome de burnout ${ }^{2}$, o sindrome de desgaste profesional, hacen parte de los principales riesgos laborales de su labor. El profesional de la enseñanza percibe y manifiesta esta situación a través de los propios síntomas de estrés, desembocando en problemáticas que aquejan hoy a todas las organizaciones educativas: un ausentismo intermitente, enfermedades laborales, aumento de la conducta violenta, intolerancia, agotamiento, despersonalización, falta de realización personal, desmotivación frente a la profesión, todas ellas problemáticas propias de las realidades escolares actuales.

\section{METODOLOGÍA}

La investigación que sustenta los resultados parciales que se presentan en este artículo, se concibió desde una perspectiva de desarrollo crítico de las ciencias, opción que se centra en la configuración de conocimiento científico socialmente pertinente, conocimiento que es validado y legitimado, además de su rigor metodológico, por la alta potencialidad transformativa de tal conocimiento en función de los contextos sociales en los cuales se configura. Así, esta investigación

2. El concepto de "Burnout" fue acuñado por Freudenberger en 1974. posteriormente Maslach y Pines (1977) lo difundieron, "dicho término se utiliza para referirse al desgaste profesional que sufren los trabajadores de los servicios humanos (educación, salud, administración pública, etc.), debido a unas condiciones de trabajo que tienen fuertes demandas sociales" (Alemañy, 2001). 
evidencia su carácter crítico en la medida en que la comprensión que se logró de la organización educativa como escenario para el desarrollo de competencias emocionales en los docentes, se constituye en fuente para la transformación de las prácticas de educación. Esta racionalidad, a la vez intencionalidad, aporta a la ampliación del campo de conocimiento del fenómeno de las competencias emocionales en Colombia y además genera espacios de reflexión en torno a la necesidad de transformación de dichas prácticas educativas en los programas de formación de docentes.

Ademas de lo anterior y desde la coordenada epistemológica de la complementariedad, se configuró un trayecto metodológico, que en la misma línea de la complejidad, permitió acercarse al fenómeno del desarrollo de competencias emocionales de manera integrada, superando las visiones parcializadas y aisladas: "el principio de complementariedad asume la necesidad de comprender realidades desde la multidimensionalidad compleja que la determina, acudiendo para ello a posturas ontológicas, epistemológicas y metodológicas..." (Murcia \& Jaramillo, 2008, p. 11).

Coherente con lo anterior, la investigación configuró una intencionalidad comprensiva que tuvo como proposito reconstruir concepciones y prácticas para el desarrollo de competencias emocionales, en el contexto de los programas de formación de docentes de las Escuelas Normales Superiores (ENS) de Colombia.

De manera particular la hermenéutica, como opción metodológica, respondió en forma adecuada al interés comprensivo. Desde esta intencionalidad se incorporó, dentro de la gama de perspectivas posibles de la hermenéutica, la lógica del "círculo hermenéutico", cuya fundamentación se realizó desde los aportes de Friedrich Daniel Ernst Schleiermacher (1997), autor original del método y en los desarrollos complementarios posteriores de Wilhelm Dilthey (2005) y Hans-Georg Gadamer (2010). De manera complementaria y atendiendo a la naturaleza compleja del problema y de la intencionalidad comprensiva, se incluyeron elementos del estudio de caso y de la teoría fundamentada.

La representación y denominación de círculo hermenéutico, según Schleiermacher (1997) expresa círculos comprensivos concéntricos que a manera de espiral denotan un proceso continuo de interconexión, comunicación y relación entre los diferentes momentos y fases interpretativas y comprensivas; en este caso en torno al desarrollo de competencias emocionales en el contexto de los programas de Formación Complementaria de las ENS que hicieron parte del estudio.

En el marco del interés comprensivo que subyace en todo proceso hermenéutico, el círculo hermenéutico aquí se justificó como axial metodológico, dado que de manera intencionada guió cada uno de los momentos y acciones a la comprensión e interpretación del fenómeno del desarrollo de competencias emocionales; buscando comprender, a partir de la articulación de todas las partes, el sentido de los estudiantes, directivos y docentes; de los textos, de los proyectos educativos, de las experiencias y vivencias, de las realidades cotidianas.

Siguiendo a Schleiermacher "la hermenéutica consiste en demostrar, a partir de las formas significativas externas, la posibilidad de hacer de la comprensión un método para reconstruir lo que el autor experimentó en el momento de elaborar su texto o discurso" (1997, p. 34). En este caso esta comprensión se realizó a partir de la exploración y análisis profundo de los perfiles y mallas curriculares de los PEI disponibles de los programas de formación complementaria de las ENS, cuestionarios aplicados a directivos de ENS, diarios de campo sobre experiencias formativas con estudiantes de ENS, grupos focales de discusión y observación directa; buscando con todo ello comprender los sentidos y significados que los actores evidencian en cuanto al desarrollo de competencias emocionales. Desde esta lógica se recorrió el camino de las pistas del lenguaje, buscando los sentidos y significados de los procesos de su desarrollo: "La comprensión como arte consiste en aplicar reglas a los textos y en una reconstrucción, re-producción o 
re-experiencia del proceso mental constructivo que ha seguido un autor en su composición" (Dilthey, 1978, p. 87).

A manera de trayecto y de manera dialéctica, la reconstitución del círculo hermenéutico general se desarrolló a través de tres momentos-procesos: momento de reconstrucción, momento de teorización y momento de configuración. En esta racionalidad, de naturaleza recursiva y simultánea, cada momento-proceso se alimentó permanentemente de las elaboraciones de los otros, conformándose según Schleiermacher (1997) círculos comprensivos concéntricos, que a manera de espiral, denotaron una dinámica continua de interconexión, comunicación y relación entre los diferentes momentos interpretativos y comprensivos. El círculo hermenéutico solo se cerró en el momento en que se lograron los propósitos investigativos planteados.

De manera complementaria al circulo hermenéutico, también se incorporaron elementos metodológicos de la teoría fundamentada; esto desde los aportes de Glasser y Straus: "El enfoque del que parte la teoría fundada es el de descubrir teorías, conceptos, hipótesis partiendo directamente de los datos, y no de supuestos a priori, de otras investigaciones o de marcos existentes" (1990, p. 248). Esta opción metodológica aportó las técnicas de análisis y la recogida de datos fundamentales para el análisis de los procesos formativos en competencias emocionales en las ENS en Colombia, haciendo posible una teorización sustantiva y rigurosa.

Como segundo elemento metodológico complementario, desde el punto de vista de la planificación de la investigación, se incorporo el uso del estudio de caso, con sus variantes instrumental y múltiple como estrategias para la recolección de la información. El primer tipo de estudio de caso empleado, el estudio de casos múltiples, permitió abarcar la mayor cantidad significativa de información, dando cuenta del fenómeno abarcando la mayor diversidad posible de contextos de nuestro país. Desde la configuración de la unidad de análisis el estudio de casos múltiple comporto la configuración del análisis de 75 PEI de ENS de 22 departamentos como muestra intencional sobre la unidad comprensiva total de 137 ENS.

El segundo tipo de estudio de caso fue el único instrumental, el cual se utilizó al seleccionar de manera intencional una ENS que sirvió de referente para la contrastación y verificación de la comprensión lograda en el círculo hermenéutico de la unidad comprensiva de caso múltiple. El caso único instrumental, según Stake (2007), permite un "proceso de indagación sistemática y crítica del fenómeno que se haya escogido, y de generación de conocimientos que se sumen a los que ya son públicos sobre el tema en cuestión" (p. 39). Esto implicó un proceso de investigación exhaustiva y desde múltiples perspectivas de la complejidad y unicidad de la ENS seleccionada, pero no como caso especial sino con el ánimo de complementar y constrastar los hallazgos y teorizaciones resultantes del análisis de los otros 75 casos de las ENS abordadas a través del análisis de los PEI.

De esta manera, la ENS seleccionada tuvo un interés secundario ya que desempeñó un papel de apoyo, de mediación instrumental, facilitando la comprensión de la caracterización y reconstrucción teórica del proceso de desarrollo de competencias emocionales en la unidad comprensiva de ENS. En esta perspectiva se pudo seleccionar cualquier otra ENS que hubiese podido proporcionar las condiciones necesarias para aportar la misma información y comprensión. En esta perspectiva la selección de la ENS para el estudio de caso instrumental se definió por la posibilidad de acceso de la investigadora.

\section{RESULTADOS}

Con base en lo planetado en la introducción previa, es evidente que las situaciones problemáticas referenciadas son fruto de un ambiente caracterizado por altas demandas emocionales, situación que encuentra su raíz en múltiples problemas, que a continuación se configuran compresivamente en cuatro escenarios problematizadores 
que hacen evidente el reclamo a la organización educativa de respuestas efectivas que impacten y desarrollen en el sentido extenso y profundo una auténtica educación emocional:

\section{- Primer escenario problematizador: la nueva estructura y condición familiar no aporta los patrones emocionales necesarios para una formación integral de calidad}

Es casi tan compleja como incontable la multiplicidad de formas que asume la estructura familiar de hoy, pero más allá de su estructura y multiplicidad, las relaciones que se generan en su interior son aún tanto más complejas. Y en esta amalgama de roles y relaciones son muchos los niños y jóvenes de hoy que no encuentran un espacio propicio para su desarrollo emocional estable.

La familia como primer agente socializador ha cambiado su configuración primaria, las nuevas condiciones de género, económicas, sociales y globales, le han impreso una dinámica que desarrolla nuevas condiciones de vida para los niños y niñas en los últimos años. La elevada tasa de separaciones y la dramática disminución en las tasas de natalidad han generado familias caracterizadas en un alto porcentaje por núcleos familiares pequeños, con altos índices de separación, dirigidos por una sola cabeza de familia, o con la ausencia de los padres a causa del fenómeno de la migración al extranjero o por la necesidad de ambos padres de laborar; todo ello ha configurado a una nueva generación en la que la soledad es la característica principal, donde los medios de comunicación y la escuela son los principales patrones.

$\mathrm{Al}$ respecto Marcela Escobar Q., en su texto Las 6 revoluciones de la familia (2005), plantea como las principales características de la familia de hoy: familias más pequeñas, postergación de la maternidad, disminución de los matrimonios, hombres redefiniendo su rol, el fenómeno de la migración; menos hermanos, menos primos, menos tíos y cada vez menos presencia de ambos padres, son las nuevas realidades. La socialización primaria ha perdido la gran fortaleza de antaño donde ya se llegaba al mundo escolar con reglas interiorizadas y aprendidas fruto de un ambiente familiar enriquecido por el afecto y la diversidad en número y acompañamiento. Los niños de hoy enfrentan el mundo de su escolaridad con escasos meses de vida y su proceso de interiorización de los esquemas emocionales y de la socialización depende casi exclusivamente de las oportunidades que le brinda el ambiente escolar.

De los antiguos tutores afectivos, los padres no enseñan por estar inmersos en el subsistir; tampoco los otros, los hermanos y los familiares, por su ausencia física. Los niños y las niñas actuales carecen de tutores afectivos, y de la asignatura más importante de la vida”. (De Zubiria, 2007, p. 329)

De este escenario problematizador surge entonces una reflexión expresada en diversos cuestionamientos: ¿están las organizaciones educativas preparadas para asumir los nuevos retos formativos que la familia ha dejado de lado?, ¿está el docente preparado para atender esta nueva generación de niños y niñas?, ¿está intencionalmente la organización educativa respondiendo a esta nueva realidad?

Si los nuevos escenarios pedagógicos proponen y claman una educación más crítica, reflexiva, propositiva para una nueva sociedad del conocimiento, la nueva dinámica familiar clama apoyo de la organización escolar en dimensiones humanas como la emocional. Psicólogos como Luria, Leontiev y Vigotsky desde hace muchas décadas enfatizaron en la importancia de que la finalidad de un sistema educativo, si corresponde a un crecimiento saludable, debe conducir a un crecimiento no solo académico, sino también emocional y social. Pero sus voces las silenciaron el predominio curricular absorbente de las "áreas mayores”, requeridas sólo para formar técnicos y profesionales. En este contexto sociocultural muy frágil en donde viven nuestros hijos, la organización educativa debe revisar su gestión, la pertinencia de su currículo, sus fines, los perfiles de sus docentes para tal tarea; en fin, su pertinencia con este nuevo papel que le correspondió. 
- Segundo escenario problematizador: la emergencia de una nueva condición de lo humano que está demandando nuevas respuestas de las organizaciones formadoras de maestros

Las nuevas condiciones de vida de las generaciones actuales, la calidad de vida de su gestación y de su niñez, las condiciones de estimulación en los primeros años, las características de alimentación y de crianza y muchos factores más, han venido desarrollando condiciones y especificidades que no se contemplaban en las generaciones anteriores; nuevos síndromes, trastornos y toda una gama de problemas afectivos han entrado a hacer parte de las nuevas condiciones que los docentes deben contemplar en la cotidianidad de sus aulas.

Multiplicidad de trastornos emocionales, inclusión, multiculturalidad, heterogeneidad... interpelan hoy las prácticas educativas de las organizaciones educativas y ponen en el escenario la necesidad de la educación emocional como dimensión fundamental de la formación integral que ofrece el sistema educativo.

Condiciones psicológicas como el TDAH (Trastorno por Déficit de Atención con Hiperactividad) son cada vez más prevalentes en las aulas de clase. Según la asociación Nacional de Psiquiatría este es un trastorno en el que intervienen tanto factores genéticos como ambientales, es un trastorno de conducta que aparece en la infancia, y que se suele empezar a diagnosticarse sobre los 7 años de edad, aunque en algunos casos este diagnóstico se puede realizar de una manera más precoz. Se manifiesta como un aumento de la actividad física, impulsividad y dificultad para mantener la atención en una actividad durante un periodo de tiempo continuado. Asociado a ello, dadas las características del trastorno, se conecta con problemas de autoestima debido a la presión social derivada de sus pautas de conducta.

En este tipo de trastornos, según estudios publicados en el 2008 por la Revista Colombiana de Neurología, la prevalencia del TDAH en Colombia oscila entre " 17,8 y el $20 \%$, sabiendo que existen dificultades para las comparación de las prevalencias obtenidas en estos trabajos, explicables por los diversos criterios diagnósticos y métodos de evaluación empleados" (Cornejo et al., 2005, p. 716). Lo que pone en cifras una realidad cotidiana que manejan los docentes de hoy, situación que muestra la dramática realidad de las aulas de clase en cuanto al manejo comportamental y emocional de estos pequeños.

Otra investigación publicada en 2009 por la Revista Nacional de Psiquiatría, además de reforzar estas cifras preocupantes hace las siguientes reflexiones:

- En el mundo, el trastorno por déficit de atención con hiperactividad (TDAH) (1) es la patología más común en la población pediátrica.

- Aunque no hay diferencias significativas en la prevalencia del TDAH entre países, los pacientes latinoamericanos con TDAH y sus familias sí tienen un perfil demográfico, sintomatológico y de respuesta a tratamiento diferentes que los hace más predispuestos a no acudir a un tratamiento a adecuado.

- El Consenso Latinoamericano de TDAH, en ciudad de México 2008, el cual produjo los algoritmos de manejo del TDAH para Latinoamérica, todo esto con el ánimo de traducir los conocimientos internacionales en propuestas para Latinoamérica.

- Se analiza la tendencia actual de manejo del TDAH de los profesionales colombianos y las limitaciones para seguir el tratamiento de estos pacientes en Colombia especialmente en el ámbito escolar. (Palacio J., Botero C., Muñoz F., Vasquez R., Carrozosa M. 2009)

Estas reflexiones hechas por profesionales de la salud a nivel internacional ponen en evidencia nuevamente la realidad de la situación y cómo desde el ámbito educativo en lugar de apoyar en la solución, por el contrario está agravándola, dado que los maestros no están preparados para asumir el trabajo con este tipo de población.

A la par del TDAH, en segunda instancia, la depresión está llegando a convertirse en un problema de salud pública; esta condición es 
una enfermedad letal que cada vez afecta más a niños, jóvenes y adultos. Datos internacionales muestran que parece cundir una moderna epidemia de depresión, que se extiende a lo largo y ancho adoptando nuevas modalidades en cada lugar del mundo. Cada nueva generación, desde principios de siglo, ha corrido riesgo mayor que la generación de sus padres de sufrir una depresión más importante, no ya tristeza, sino un desinterés paralizante, desaliento y autocompasión, más una abrumadora desesperanza, en el curso de su vida (Goleman, 2005, p. 134).

La Organización Mundial de la Salud (OMS) en octubre de 2012 celebró el aniversario $20^{\circ}$ del Día Mundial de la Salud e hizo un llamado mundial a la atención y desestigmatizacion de la depresión y otras enfermedades mentales comunes hoy día. En el estudio publicado por la OMS en ésta fecha se estima la prevalencia de la depresión en los países de la organización en un $5 \%$, con un número aproximado de 350.000 .000 de personas de todas las edades que la padecen.

Es imposible no preguntarse el porqué de la creciente oleada de pacientes diagnosticados con depresión que se encuentran día a día en todos los consultorios médicos y psicológicos del mundo. Este organismo también pronostica que para el año 2020, será la patología que causará más pérdida de años de vida saludable, sólo superada por las dolencias cardiovasculares.

A la par con la depresión, el suicidio como comorbilidad de esta, según lo plantea la Organización Mundial de la Salud, es un problema de salud mental en el mundo, cada vez más las nuevas generaciones encuentran en él la salida a sus problemas frente a la incapacidad de auto-administrar sus propias condiciones y emociones. Para muchas personas hoy acudir al suicidio parece ser la única manera de salir de las situaciones conflictivas, mostrando así como la humanidad es cada vez más vulnerable y cuenta con menos herramientas para responder a las presiones de su contexto. La OMS, en este informe, dice que 1.000.000 de personas se suicidan cada año en todo el mundo, y cada día 800 personas se suicidan en el mundo por culpa de la depresión.
Triste realidad en un mundo donde mueren más personas por suicidio que por los conflictos bélicos. Y frente a estas estadísticas en crecimiento ya la realidad no nos convoca frente a casos especiales sino que nos apremia frente a realidades que se salen de la cobertura de los procesos formativos tradicionales del aula. En agosto 3 de 2010 fue publicado un estudio elaborado por la Liga Colombiana por la Vida y contra el Suicidio en los colegios de la ciudad de Bogotá, en donde se ratifica el estudio de la Organización Mundial de la Salud, concluyendo que uno de cada dos estudiantes de bachillerato en Colombia tiene un plan preciso para quitarse la vida y uno de cada seis lo ha intentado sin éxito. Recalcando que la alarmante estadística develaba una situación que es preocupante en un país donde no existe una política concreta que apunte a mitigar esta problemática.

Frente a esta realidad el compromiso con esta nueva dinámica humana interpela la responsabilidad de la nueva educación, y este compromiso no debe pensarse frente a niños "fuera de lo normal”; el compromiso es frente a una generación más vulnerable en lo emocional, frente a una condición humana cambiante que requiere de más espacios formativos, frente a una dinámica social que requiere de competencias emocionales más pertinentes con las nuevas realidades del mundo de la vida de hoy.

Pero es necesario aclarar que toda esta reflexión no pretende circunscribir la necesidad de problematizar esta investigación en el marco de las patologías, por el contrario, la formación emocional se refiere a toda la condición de lo humano, se refiere al día a día del aula y de la organización educativa en su constante devenir de relaciones e interacciones. La alfabetización emocional (Goleman, 2011) es un imperativo de la educación actual; nuestros niños y jóvenes requieren una formación integral que los prepare para la realidad que les correspondió vivir y en este sentido el desarrollo emocional es un tema crucial que les permitirá asumir competentemente su vida adulta.

Reflexionar sobre la educación emocional de nuestros estudiantes y de nuestros docentes es 
pensar en el reto de una educación para la vida, auto-administrar la vida emocional es la clave del saber vivir bien, es tener la capacidad de vivir en un mundo reflexivo e intencional donde seamos capaz de ser conscientes de nuestra vida emocional, donde se reconoce al otro y se es capaz de trabajar y convivir con él a través de la empatía y el trabajo en equipo, donde se tiene la capacidad de automotivación para superar la adversidad a través de la autoeficacia y la resiliencia.

Desde este escenario problematizador surgen entonces preguntas frente a la calidad de la educación y la manera cómo esta responde a este reto formativo: ¿se están generando espacios formativos para los docentes en los cuales se visibilice y se les de estrategias para desarrollar las competencias emocionales necesarias?, ¿qué están haciendo las organizaciones educativas para interpelar la realidad emocional que afecta y determina los resultados académicos?

- Tercer escenario problematizador: las nuevas realidades y desafíos que imponen la sociedad y los medios de comunicación a la formación emocional

Como tercer escenario, se plantea que la sociedad actual enfrenta a nuestros docentes a una serie de fenómenos culturales, políticos y sociales que traspasan las fronteras del aula, interpelando la cotidianidad misma de la organización educativa al ser ella misma una reproducción micro de esa misma sociedad. Uno de esos fenómenos es la violencia en sus diferentes manifestaciones y la visualización y exacerbación que de ella hacen los medios de comunicación.

Uno de los centros especializados para el estudio de la violencia en Colombia es el Instituto de Investigación y Desarrollo en Prevención de Violencia y Promoción de la Convivencia Social -CISALVA- de la Universidad del Valle (Cali, Colombia). En una investigación publicada en el portal de dicha institución en abril de 2008, el CISALVA analiza como "la violencia de tipo político generada por la guerrilla, paramilitares y ejército, a pesar de ser la más publicitada, la que más impacto económico genera y la causante de 1’500.000 desplazados en el país, sólo suma entre el $20 \%$ y $30 \%$ del total de muertes violentas en nuestro país” (Tavera, 2008). Igualmente en muchas ciudades, contrario a lo que usualmente se cree, la violencia generada por el crimen organizado tampoco es la que más víctimas causa. El fenómeno cada vez se agrava con la prevalencia de otro tipo de violencias de orden intrafamiliar, de género, por patrones establecidos por los medios de comunicación, matoneo entre otros. Estas violencias cotidianas son tan graves que se las considera una epidemia puesto que afectan amplios núcleos poblacionales.

Somos fruto de una cultura, pero como actores culturales tenemos la responsabilidad no solo de reproducirla sino de reconstituirla y mejorarla y en este sentido el interrogante es como educadores: ¿qué estamos haciendo para tal fin?, ¿cómo está reconstituyendo y transformando esta realidad social el escenario escolar?, ¿o por el contrario, la escuela es a su vez escenario de violencia e irreflexión?, ¿está la realidad de la violencia interpelando la intencionalidad y racionalidad de las prácticas educativas, o seguimos perpetuando una educación a espaldas de una realidad que aunque nos afecta no la queremos ver?

El espacio de la escuela como escenario cultural es el llamado a transformar desde la reflexión y la autonomía esta cultura de violencia y dar cuenta de mejores hombres y mujeres para un futuro mejor. El pasado no pudo ser mejor o peor, las condiciones han cambiado y ahora como educadores nos compete una responsabilidad social más allá de lo cognitivo en un escenario de lo humano integral para construir un mundo mejor.

Pero además de la visualización y apología que de la violencia hacen los medios de comunicación, la influencia de estos están impactando con gran poder la construcción de imaginarios colectivos. Sí es cierto que las razones que impulsan a los niños a ver televisión, películas o utilizar juegos interactivos son pasar el tiempo, aprender, sentirse acompañados, escapar, sentirse estimulados y reflejarse, criterios fundamentales para un sano desarrollo afectivo y cognitivo; sin embargo, no por ello se puede desestimar la influencia negativa que a su vez éstos están dejando en 
el inconsciente colectivo y como este poder ha sido manipulado por la publicidad y la sociedad de consumo en aras de intereses económicos y particulares que en nada tienen que ver con el bienestar de la población infantil.

En una reciente encuesta realizada en EEUU a 30.000 personas y publicada en la revista el Psichology Today (2012) se destaca que un $93 \%$ de las mujeres y un $82 \%$ de los varones interrogados están preocupados por su apariencia y trabajan para mejorarla, situación que de por sí es la evidencia de los altos índices de trastornos alimentarios en el mundo de hoy. "Desde muy jóvenes, expresa el Dr. Olkies en el artículo, las representantes del sexo femenino están sometidas a una gran presión para satisfacer cierto ideal de belleza que la moda impone con rigor y sin consideraciones. Para los varones esto ocurre en menor medida”. Esta especie de dictadura de la moda exige máxima delgadez, la cual debe ser alcanzada sin reparar en los costos, desde la pantalla y las revistas desfilan diariamente los máximos exponentes del "genero": si te encuentras entre ellos estas "in", en caso contrario, se pasa a formar parte de los "out". Esta realidad somete a los jóvenes y niños y niñas a una sobre carga emocinal desde edades muy tempranas.

Se ha de tener en cuenta que los medios de comunicación en la actualidad juegan un papel importante en la socialización y estimulación de los niños y niñas, y no por ello se puede negar la influencia negativa de éstos en esos mismos procesos de desarrollo, ya que presentan conductas imitables, proporcionan imágenes para provocar determinadas acciones y son el medio por el que los niños se familiarizan con los valores de la sociedad de ocio y de consumo.

La sobreestimulación de los pequeños cada vez interpela más al aula escolar, los métodos y las herramientas cada vez tienden a ser menos motivantes y cada vez es más difícil para los docentes hablar el mismo idioma de los jóvenes. En los corredores y aulas cada vez se imitan más comportamientos estereotipados de películas y pandillas de la pantalla y cada vez es más difícil para los jóvenes encontrar su identidad en un mar de propuestas.

Este escenario entonces plantea nuevamente la pregunta por el papel de las organizaciones educativas y del maestro: iestá la organización educativa dando los elementos necesarios para que los estudiantes reciban con autonomía y reflexión en esta sociedad de la información?, ¿está la organización educativa replicando una cultura de violencia o por el contrario está construyendo nuevos escenarios de convivencia pacífica?

\section{- Cuarto escenario problematizador: las difi- cultades y retos para la formación docente, en particular en el ámbito de las emociones}

El aula de clase y la organización escolar, de manera micro, reproducen la sociedad de la cual hacen parte; en este sentido los escenarios problematizadores descritos anteriormente hacen parte de la cotidianidad del docente de su realidad como persona, como ciudadano, como sujeto de aprendizaje.

En esta perspectiva, las organizaciones encargadas de la formación de los maestros en nuestro país, las Facultades de Educación y las Escuelas Normales, están llamadas a revisar sus propuestas formativas para responder a las nuevas dinámicas de la sociedad actual, pero más aún las necesidades de sus docentes en formación. En un mundo donde la sociedad del conocimiento multiplica y redimensiona el conocimiento permanentemente ya no es suficiente la formación de docentes con base en el conocimiento, porque nunca estarán actualizados. En este nuevo escenario es indispensable la formulación de unas competencias que permitan al docente, a partir del conocimiento de las bases teóricas de su saber disciplinar, la pedagogía y la didáctica, avanzar en la gestión de la información en su campo de estudio a través de habilidades, destrezas y actitudes que le permitan desempeñarse eficiente y éticamente en el mundo que le correspondió vivir. Como lo afirman Fernández et al. (2009, p. 43), es necesario defender el valor de la educación emocional en las aulas no solo de 
educación inicial sino también en las aulas de formación de maestros como pilar fundamental en la construcción de una nueva educación.

Es momento de hacer un alto en el camino y evaluar cómo se está formando a nuestros docentes, y qué tipo de organizaciones educativas se están configurando a través de ellos y qué tipo de paradigma educativo está respondiendo a las realidades del mundo de hoy. No sería justo como sociedad exigir al docente un tipo de educación de la cual no ha sido sujeto de formación, de la cual no tiene las bases necesarias para asumirla.

El aula de clase es un escenario social altamente exigente para el docente y no se le está preparando con las herramientas emocionales, didácticas y pedagógicas necesarias para asumir el reto. Si se quiere una mejor calidad de la educación que responda a esta nueva humanidad que correspondió educar, solo se hará con docentes altamente calificados no solo en lo disciplinar, sino también en lo didáctico y lo humano: "en definitiva, el profesorado trabaja con los niveles de competencia social y emocional que ha podido desarrollar de un modo casi intuitivo a lo largo de su proceso de socialización. Las carencias que aparecen en la práctica profesional no son achacables a que los docentes sean defectuosos, sino que son debidos fundamentalmente a la precariedad o ausencia de una formación específica en el terreno de las inteligencias intrapersonal e interpersonal (Palomero y Fernández, 2009, p. 147).

\section{CONCLUSIÓN}

Desde los escenarios de problematización anteriores quedan expuestas algunas de las necesidades formativas actuales a las que la organización educativa debe responder integralmente. Para poder responder a ese reto es necesario iniciar por la tarea de implementar en los programas de formación de docentes esta dimensión tan importante.

Este reto dada su naturaleza compleja como objeto de estudio adquiere una doble condición formativa, epistémica y empírica, que siguiendo a Sánchez (2010) la ...dimensión epistémica alude al conjunto de principios, conceptos, planteamientos y elaboraciones de naturaleza teórica que constituyen y delimitan el objeto de estudio y la dimensión empírica se refiere a la red de prácticas específicas y concretas, a situaciones casuísticas y específicas que se evidencian objetualmente en un contexto social determinado. (Sánchez, 2010, p. 17)

Es así como los docentes deben tener una formación epistémica, teórica, en cuanto a la naturaleza de la dimensión emocional como objeto de estudio, a fin de entender los desarrollos teóricos que al respecto del tema constituyen el estado de la cuestión al respecto, pero a las vez y complementario de ello deben ser sujetos de desarrollo de competencias emocionales, ya que como sujetos educables en sí mismos requieren desarrollar la dimensión emocional que los hace ser presencia viva de una persona competente emocionalmente.

En esta línea de pensamiento Fernández (2009) plantea que los docentes deberían tener una formación en el ámbito de la educación emocional, estar preparados a nivel teórico y desarrollar competencias personales:

...que les ayude a ser personas más conscientes de sí mismas, más maduras y equilibradas, para que así puedan influir positivamente en niños y adolescentes, y para que desde su propia solidez personal les ayuden a crecer y a ser ellos mismos, desplegando todas sus potencialidades. (Fernández et al, 2009 p. 36)

Dada la tradición científica y derivada de ella, la tradición pedagógica predominante en el proceso de escolarización en nuestro país, los procesos educativos han estado marcados por un predominio de lo cognitivo sobre cualquier otra dimensión del ser humano. A través de la historia se han subvalorado las otras dimensiones del ser como procesos susceptibles de ser intervenidos educativamente; es así como lo físico, emocional, moral, social, político; entre otras han sido, y continúan siendo, competencias excluidas de las aulas escolares y los programas de formación docente no son la excepción. Esta situación se constituye en un argumento más para generar 
un escenario problematizador que justifica el acercamiento a una educación más integral, en este caso, una educación que propenda por la formación de la emocionalidad como dimensión fundamental del ser humano.

Estos escenarios problematizadores son una evidencia de cómo los maestros que hacen parte de nuestro sistema educativo no están respondiendo ante las realidades sociales más apremiantes de nuestra sociedad; de como la organización educativa ha estado de espaldas a las demandas que la sociedad le hace de manera apremiante. Pero esta visión del deber ser de la educación deja por fuera otra consideración de vital importancia como lo es el bienestar de los docentes, quienes al hacer frente sin las herramientas requeridas a las múltiples dinámicas de los procesos educativos de su cotidianidad y a la permanente presión social por resultados, no están preparados para asumirlas y también sufren las consecuencias en su salud física y mental.

En estos escenarios problematizadores se enfatiza que el campo laboral docente tiene la característica de ser un ejercicio profesional de alto desgaste emocional: como profesión de constante interacción, la docencia connota procesos emocionales, físicos y mentales complejos; por eso, la formación en competencias emocionales ejerce efectos beneficiosos para el profesorado a nivel preventivo.

Es decir, la capacidad para razonar sobre las emociones, percibirlas y comprenderlas, como habilidad intrínseca del ser humano, implica, en último término, el desarrollo de procesos de regulación emocional que ayudarían a moderar y prevenir los efectos negativos del estrés docente a los que los profesores están expuesto diariamente. (Extremera \& FernandezBerrocal, p. 4)

La Fundación Médico Preventiva (FMP) es una organización privada que ha sido responsable de prestar servicios de salud y administrar riesgos profesionales a los docentes oficiales del Departamento de Antioquia, Colombia. La FMP reveló que para el primer trimestre del año 2004 el síndrome de burnout ya aparecía entre las 5 enfermedades más prevalentes en el personal docente del Departamento de Antioquia. En estos estudios según Cabreras y Gabriel (2006) el burnout es un cuadro progresivo aparece por efecto de exposición a estresores laborales y que se expresa con agotamiento emocional, despersonalización y bajo sentido de logró junto con alto índice de incapacidad laboral, física y psicológica.

En este síndrome tienen un papel importante la presión del trabajo y el ambiente físico que rodea al maestro, que en la actualidad en el sector oficial y más en la región Caribe connota plantas físicas inadecuadas y sin las condiciones propias para un proceso de calidad en donde el promedio por aula es de 45 a 50 estudiantes, por lo que se puede definir también como un estrés laboral prolongado, no resuelto, que demanda atención especializada en las dimensiones médica, psicológica y organizacional.

Un estudio publicado en 2010 por la Revista Universitas Psychologica de Cali, Colombia, cuyo objetivo fue establecer los factores asociados al síndrome de burnout en docentes en instituciones educativas formales privadas y públicas de la ciudad de Cali, Colombia, donde a pesar que se llega a la conclusión que la prevalencia es moderada, se muestra el resultado de un $15 \%$ de los docentes de la muestra que ya presentan niveles moderados del síndrome, es decir ya son diagnosticados con la sintomatología inicial y un $48 \%$ presentan agotamiento emocional que es una de las sintomatologías precursoras del síndrome.

A pesar del rastreo bibliográfico no se pudieron encontrar estudios al respecto de esta dimensión de la docencia en ciudades de la región Caribe, situación que denota igualmente la falta de atención que se le da a esta problemática.

Frente a estos escenarios que plantean los retos que deben asumir los docentes y también que plantean el precio que están pagando por su incapacidad para enfrentarlos surgieron preguntas que interpelaron el trayecto de esta investigación: ¿cómo asumen estas nuevas dimensiones las Facultades de Educación?, ¿cómo se prepara al docente para responder a esta realidad?, ¿sigue 
imperando solo la preocupación por la formación disciplinar del docente dejando de lado todo lo necesario para enfrentar este panorama social?

En consecuencia, y mirando la realidad de nuestros docentes, se argumenta que en la actualidad la formación docente no solo debe incluir la dimensión disciplinar de su objeto de estudio, la dimensión didáctica de su enseñabilidad y la pedagogía que la sustenta epistemológicamente, sino que a su vez debe poseer los conocimientos y actitudes que lo preparen para asumir un papel activo en el desarrollo emocional de sus estudiantes de manera intencional y a través de su propio estilo de enseñar: "En consecuencia, consideramos que el profesor no sólo tiene la obligación de conocer las materias que explica y los correspondientes métodos de enseñanzaaprendizaje, sino que debe ocuparse también de comprender a los estudiantes, de interesarse por su espacio vital, por su mundo de la vida: es decir, por lo que viven, por sus emociones, por lo que sienten, por lo que piensan" (Fernández, Palomero, \& Teruel, 2009 p. 34). En este sentido el reto se convierte no solo en una tarea de la formación inicial de los maestros, sino que se convierte en el reto de las organizaciones educativas en torno a la formación continua de sus maestros. Ser un maestro eficaz hoy en día no solo requiere la actualización y ampliación del campo disciplinar con el continuo avance de la sociedad del conocimiento, requiere también renovarse permanentemente como ser humano que siente, que se enfrenta a nuevos retos sociales y humanos que le exigen altas dosis de desgaste emocional. En este contexto, la educación emocional es un elemento fundamental para el profesorado.

El mundo de hoy plantea para las nuevas generaciones un reto de sobrevivencia, reto que no debe ser asumido por ellos únicamente, sino también por las generaciones encargadas de educarlos,

"la nueva orientación que invita a llevar la alfabetización emocional a la escuelas, convierte las emociones y la vida social en temas en sí mismos, en lugar de tratar estas facetas apremiantes de la vida cotidiana del niño como estorbos sin importancia..." (Goleman, 2005, p. 303)
Para que estos cambios que se requieren en las organizaciones educativas es fundamental la labor de los docentes. El como actor principal juega un papel primordial dado que más allá de hablar de un fenómeno educativo, hablamos de seres humanos "docentes" que hacen vida ese fenómeno; por eso desde la perspectiva de este texto se propende por una formación más integral de ellos, lo que implícitamente conlleva afectar el fenómeno total; en este sentido y en palabras de Messina (1999), se reconoce el papel estratégico de los docentes en las transformaciones educativas.

Para cumplir este encargo encargo estratégico, el docente de hoy debe contar con un perfil integral que le permita asumir las demandas de la nueva sociedad. Una de las competencias de ese perfil son las emocionales, que como en el presente texto se afirma son estratégicas frente a los requerimientos del perfil laboral, dado el tipo de labor que desempeñan, que es en sí misma una labor de interacción humana y por ende cargada de emocionalidad.

Esta condición estratégica de las competencias emocionales docentes tiene muchas consideraciones. Al respecto es necesario reiterar que "enseñar es un acto emocional por acción o por omisión, por diseño o por defecto” (Extremera yFernández Berrocal, 2003, p497), razón por la cual no podría hablarse de un proceso educativo de calidad que deje por fuera esta dimensión.

En esta perspectiva, el docente enseña no solo contenidos teóricos, el docente como persona es un factor determinante en el aprendizaje, como patrón de comportamiento, como modelo, como referente. A la hora de influir en las actitudes y en los hábitos de los estudiantes es la personalidad del profesor la que en últimas forma a través del ejemplo y de la experiencia cotidiana.

La dinámica de la sociedad del conocimiento ha variado el papel de docentes de hoy, las tecnologías de la información y la comunicación han desplazado la función poseedora del conocimiento por parte del docente, facilitando a los estudiantes el acceso al saber por medios más expeditos y atractivos; el saber ya no reside 
exclusivamente en la organización escolar y en el docente ya no reside la responsabilidad de albergarlo. En esta nueva sociedad del conocimiento el rol del docente se ha convertido en un papel más dinámico y dialógico, el cual se centra en la potencialización de competencias, habilidades y destrezas para hacer uso de la información en un contexto de sentido. En esta dinámica, la dimensión de lo humano retoma especial importancia y el factor emocional entra a jugar un papel estratégico, "por la obsolescencia del conocimiento y las nuevas tecnologías de la información y la comunicación, el rol del profesor cambia, pasando de la enseñanza a la relación emocional de apoyo" (Bisquerra, 2011, p. 23). Para ello es imperante la formación de nuevas generaciones de docentes que asuman este reto.

A partir de los procesos vividos por la sociedad colombiana a lo largo del siglo XX con su transito hacia patrones familiares caracterizados cada vez más por la presencia de menos figuras de formación afectiva, en muchos casos siendo la nueva estructura incapaz de proporcionar patrones efectivos de crianza y fortaleciendo el rol de terceros que ayudan a la crianza de los hijos. En este sentido el rol de la organización escolar y en ella en la del docente, se configura actualmente hacia un perfil socializador y de alfabetización emocional de apoyo que ya no es exclusivo de la familia.

Paralelo al fenómeno familiar, las tecnologías de la información y la comunicación irrumpieron con fuerza como nuevos agentes socializadores. A través de estos nuevos patrones culturales y modelos propios de la aldea global se han gestado nuevas generaciones más vulnerables emocionalmente, situación que no puede ser desestimada por los sistemas educativos: "cada nueva generación, desde principios de siglo, ha corrido un riesgo mayor que la generación de sus padres de sufrir una depresión más importante, no ya tristeza, sino un desinterés paralizante, desaliento y autocompasión, más una abrumadora desesperanza, en el curso de su vida” (p. 89).

Además del imperativo de la nueva sociedad del conocimiento, las nuevas condiciones de diversidad de las aulas, la heterogeneidad de estilos y necesidades educativas, el contexto social y familiar, exigen del docente intervenciones y procesos que en la mayoría de las casos no está preparado para asumir; es entonces necesario diseñar planes y programas de formación docente para suplir estas necesidades didácticas y de desempeño actitudinal. En esta perspectiva la educación emocional es una parte fundamental de la formación integral de la personalidad y por ello debería estar presente en cada proyecto educativo de las organizaciones educativas y muy especialmente en aquellas organizaciones encargadas de la preparación de todos los profesionales docentes. La realidad, sin embargo, es que está prácticamente ausente de los actuales planes de estudio y que, mirando al futuro, el panorama no es muy alentador (Fernández, Palomero, \& Teruel, 2009, p499).

Adicionalmente como bien lo afirma Palomero (2009), en definitiva el profesorado trabaja con los niveles de competencia social y emocional que ha podido desarrollar de un modo casi intuitivo a lo largo de su proceso de socialización. Las carencias que aparecen en la práctica profesional no son atribuibles a que los docentes no posean las competencias necesarias para el ejercicio de su docencia, sino a la precariedad o ausencia de una formación específica en el terreno de las competencias emocionales.

Además de su función social y de docencia en el aula, más allá del perfil laboral, el docente es un ser humano que tiene la característica de desempeñarse en un rol muy desgastante; como profesión de constante interacción la docencia connota grandes desgastes emocionales, físicos y mentales y la formación en competencias emocionales ejerce efectos beneficiosos para el profesorado. Es decir, la capacidad para razonar sobre las emociones, percibirlas y comprenderlas y regularlas ayuda a moderar y prevenir los efectos negativos del estrés al que los profesores están expuestos diariamente. La prevalencia del síndrome de burnout o síndrome del profesor quemado, aún en Colombia no es tenida en cuenta como variable de la calidad educativa, pero cada vez más las organizaciones aquejan 
problemas laborales, ausentismos, conflictos permanentes y actitudes docentes que van en detrimento de la calidad de la educación.

Las organizaciones educativas no cuentan con los medios, ni los docentes cuentan con las herramientas para hacerle frente a este fenómeno propio de su profesión; estamos asistiendo a un tránsito en la naturaleza de la labor docente y no podemos simplemente quedarnos de manos cruzadas a ver como nuestros docentes se "queman” en su desempeño laboral, por ello también la importancia de la educación emocional en los docentes.

A partir de estas afirmaciones y siguiendo el reto impuesto por la nueva sociedad, las organizaciones educativas están llamadas a evaluar su labor formadora y el reto inicia con superar el paradigma imperante que da prevalencia a la formación exclusivamente cognitivista, incorporando la educación en la dimensión emocional.

\section{REFERENCIAS BIBLIOGRÁFICAS}

Bisquerra, R. (2011). Educación emocional y bienestar. Barcelona España: Wolters Kluwer.

Castro, B. (2012). la organización Educativa: Una aproximación desde la complejidad. Estudios Pedagógicos Universidad Austral de Chile, 78-93.

Cornejo J.W., Sanchez Y., Carrizosa J.,Grisales H., Castillo Parra H., Holguin J. (2005). Prevalencia del transtorno deficitario de la atención e hiperactividad en niños y adolescentes Colombianos. Revista de Neurología. 40 (12) 716-721 Medellin Colombia.

De Zubiria, M. (2007). La afectividad humana. Sus origenes, sus instrumentos y operaciones. Cómo medirla con escalas y afectográmas. Bogotá (Colombia): fundación Internacional de Pedagogía Conceptual.

Dilthey, W. (1978). Obras. México: Fondo de cultura Económica.

Escobar, Q. M. (2005). Las seis revoluciones de la Familia. Santiago de Chile. Editorial Valdivia.
Extremera, N., \& Fernandez-Berrocal, P. (2005). la importancia de desarrollar la Inteligencia Emocional en el profesorado. Revista Iberoamericana de Educación, 1-10

Fernandez, M. d., Palomero Pescador, J. e., \& Teruel melero, M. d. (2009). El desarrollo socioafectivo en la formación inicial de maestros. REIFOP Revista Interuniversitaria de Formacion del profesorado 12 (1) (12 (1)), 33-50.

Glasser, B., \& Strauss, A. (1990). El descubrimiento de la teoria Fundamentada. Barcelona: Imperio.

Goleman, D. (2005). La inteligencia emocional. Mexico D.F.: Zeta.

Messina, G. (Enero-Abril de 1999). Investigación en formación docente: un estado del arte en los noventa. Revista Iberoamericana de Formación Docente, 90-99.

Morin, E. (1998). El método. La vida de la vida. Madrid, España. Editorial Cátedra.

Murcia, N., \& Jaramillo, L. G. (2008). Investigación cualitativa. "La complementariedad". Armenia Quindio: Kinessis. Colección Investigación.

Palacio J., Botero C., Muñoz F., Vasquez R., Carrozosa M., Hallazgos de una encuesta sobre la experiencia del manejo clínico del TDAH. Revista Colombiana de Psiquiatria (2009) V38 supl. 1 Octubre. Bogota Colombia.

Palomero, P. \& Fernandez-Berrocal, P. (2009). Desarrollo de la competencia social y emocional del profesorado: una aproximación desde la psicología humanista REIFOP. Revista Electrónica Interuniversitaria de Formación del Profesorado(12), 145-153.

Sanchez Buitrago, J. (2009). Una perspectiva emergente para concebir la organizacion educativa como fundamento para transformar su desarrollo. En J. Elias Caro, \& J. E. Caro (Ed.), Cultura y Sociedad (págs. 191 - 212). Santa Marta D.T.C.H. , Colombia: Editorial Universidad del Magdalena.

Sanchez Buitrago, J. (2010). Hacia un paradigma emergente de la planeacion. Resignificación desde las Instituciones universitarias. Santa Marta D.T.C.H.: Editorial Universidad del Magdalena.

Schleiermacher, F. (1805). El Esbozo. (V. G. 2000, Trad.) Madrid, España: Editorial Gredos: Madrid, 1997.

Stake, R. (2007). Investigación y estudio de casos (Cuarta ed.). Madrid, España: Ediciones morata. 